\title{
Blocking optimal arborescences
}

\author{
Attila Bernáth* Gyula Pap ${ }^{\dagger}$
}

October 1, 2018

\begin{abstract}
The problem of covering minimum cost common bases of two matroids is NP-complete, even if the two matroids coincide, and the costs are all equal to 1 . In this paper we show that the following special case is solvable in polynomial time: given a digraph $D=(V, A)$ with a designated root node $r \in V$ and arc-costs $c: A \rightarrow \mathbb{R}$, find a minimum cardinality subset $H$ of the arc set $A$ such that $H$ intersects every minimum $c$-cost $r$-arborescence. By an $r$-arborescence we mean a spanning arborescence of root $r$. The algorithm we give solves a weighted version as well, in which a nonnegative weight function $w: A \rightarrow \mathbb{R}_{+}$(unrelated to $c$ ) is also given, and we want to find a subset $H$ of the arc set such that $H$ intersects every minimum $c$-cost $r$-arborescence, and $w(H)=\sum_{a \in H} w(a)$ is minimum. The running time of the algorithm is $O\left(n^{3} T(n, m)\right)$, where $n$ and $m$ denote the number of nodes and arcs of the input digraph, and $T(n, m)$ is the time needed for a minimum $s-t$ cut computation in this digraph. A polyhedral description is not given, and seems rather challenging.
\end{abstract}

Keywords: arborescences, polynomial algorithm, covering

\section{Introduction}

Let $D=(V, A)$ be a digraph with vertex set $V$ and $\operatorname{arc}$ set $A$. A spanning arborescence is a subset $B \subseteq A$ that is a spanning tree in the undirected sense, and every node has in-degree at most one. Thus there is exactly one node with in-degree zero, we call it the root node. Equivalently, a spanning arborescence is a subset $B \subseteq A$ with the property that there is a root node $r \in V$ such that $\varrho_{B}(r)=0$, and $\varrho_{B}(v)=1$ for $v \in V-r$, and $B$ contains no cycle. An arborescence will mean a spanning arborescence, unless stated otherwise. If $r \in V$ is the root of the spanning arborescence $B$ then we will say that $B$ is an $r$-arborescence. Arborescences form a natural directed variant of spanning trees and they arise in applications where we want to broadcast information in a network that contains directed connections, for example wireless devices, too.

The Minimum Cost Arborescence Problem is the following: given a digraph $D=(V, A)$, a designated root node $r \in V$ and a cost function $c: A \rightarrow \mathbb{R}$, find an $r$-arborescence $B \subseteq A$ such that the $\operatorname{cost} c(B)=\sum_{b \in B} c(b)$ of $B$ is smallest possible. Fulkerson [8] has given a two-phase algorithm solving this problem, and he also characterized minimum cost arborescences. A natural variant of the Minimum Cost Arborescence Problem is where the root is not fixed, i.e. find an arborescence in a digraph that has minimum total cost. A simple reduction shows that this global minimum cost arborescence problem is equivalent with the Minimum Cost Arborescence Problem stated above. Kamiyama in [12] raised the following question.

Problem 1 (Blocking Optimal $r$-arborescences). Given a digraph $D=(V, A)$, a designated root node $r \in V$ and a cost function $c: A \rightarrow \mathbb{R}$, find a subset $H$ of the arc set such that $H$ intersects every minimum cost $r$-arborescence, and $|H|$ is minimum.

The minimum in Problem 1 measures the robustness of the minimum cost arborescences, since it asks to delete a minimum cardinality set of arcs in order to destroy all minimum cost $r$-arborescences. One might ask why we fix the root of the arborescences that we want to cover. The problem of finding the minimum number of arcs that intersect every (globally) minimum cost arborescence can be reduced to Problem 1 1as follows. Add a new node $r^{\prime}$ to the digraph and connect $r^{\prime}$ with every old node by high cost and high multiplicity arcs. Then

*MTA-ELTE Egerváry Research Group, Department of Operations Research, Eötvös University, Pázmány Péter sétány 1/C, Budapest, Hungary, H-1117. Research supported by the Hungarian Scientific Research Fund (OTKA, grant number K109240), and by the ERC StG project PAAl no. 259515. Part of the research was done while the author was at Warsaw University, Institute of Informatics, ul. Banacha 2, 02-097 Warsaw, Poland. E-mail: bernath@cs.elte.hu.

${ }^{\dagger}$ MTA-ELTE Egerváry Research Group, Department of Operations Research, Eötvös University, Pázmány Péter sétány 1/C, Budapest, Hungary, H-1117. Supported by the Hungarian Scientific Research Fund (OTKA, grant number K109240). E-mail: gyuszko@cs.elte.hu. 
minimum cost arborescences in this new instance will be necessarily rooted at $r^{\prime}$, they will only contain one arc leaving $r^{\prime}$ by the high cost of these arcs, and an optimal arc set intersecting these will not use the new arcs because of their high multiplicity.

One interpretation of Problem 1 is that we want to cover the minimum cost common bases of two matroids: one matroid being the graphic matroid of $D$ (in the undirected sense), the other being a partition matroid with partition classes $\delta^{i n}(v)$ for every $v \in V-r$. The problem of covering minimum cost common bases of two matroids is in general NP-complete, even if the two matroids coincide, and the costs are all equal to 1. That is, given a matroid with an independence oracle, it is NP-complete to find a minimum cut (where a cut in a matroid is an inclusionwise minimal subset that intersects every base). For a proof, see for example Theorem 2.1 in [2] and apply it to the dual matroid. In [10] we investigated the problem of covering all minimum cost bases of a matroid. We have given an algorithm that solves the problem in polynomial time, provided that we can find a minimum cut in certain minors of the matroid at hand. This includes the class of graphic matroids, that is the following problem is polynomial time solvable: given an undirected graph and a cost function on its edges, find a minimum size set of edges which intersects every minimum cost spanning tree. In fact this is a not too hard exercise for the interested reader, however the details can be found in [10].

Kamiyama [12] solved special cases of Problem 1 and he investigated some necessary and sufficient conditions for the minimum in this problem. In this paper we give a polynomial time algorithm solving Problem 1 . In fact, our algorithm will solve the following, more general problem, too.

Problem 2. Given a digraph $D=(V, A)$, a designated root node $r \in V$, a cost function $c: A \rightarrow \mathbb{R}$ and a nonnegative weight function $w: A \rightarrow \mathbb{R}_{+}$, find a subset $H$ of the arc set such that $H$ intersects every minimum c-cost r-arborescence, and $w(H)$ is minimum.

The rest of this paper is organized as follows. In Section 2 we describe related work. In Section 3 we give variants of the problem based on Fulkerson's characterization of minimum cost arborescences. These variants are all equivalent with Problem 1 as simple reductions show, so we deal with the variant that can be handled most conveniently. In Section 4 we solve the special case of covering all arborescences. This is indeed a very special case, but the answer is very useful in the solution of the general case. Section 5 contains our main result broken down into two steps: in Section 5.1 we introduce a min-min formula that gives a useful reformulation of our problem, and we give two proofs of this min-min formula (in Section 5.1 and in Section 5.2). Finally -after introducing some essential results and techniques in Section 5.3 we give a polynomial time algorithm solving Problems 1 and 2 in Section 5.4. We analyze the running time of this algorithm in Section 5.5. In Section 6 we give further remarks: we reduce Problems 1 and 2 to the problem of covering the common bases of a graphic matroid and a partition matroid, which might be a direction for further research.

\section{Related work}

The problem that we consider (Problem 1 and its weighted version, Problem 2) is a typical covering problem: given a hypergraph on ground set $S$ with hyperedge set $\mathcal{E} \subseteq 2^{S}$, find a minimum size subset $F$ of $S$ that intersects every hyperedge in $\mathcal{E}$. In Problem 1 the ground set $S$ is the set of the arcs of a digraph, and $\mathcal{E}$ is the set of optimal $r$-arborescences. Every hypergraph covering problem defines a packing problem in a natural way: given a hypergraph with hyperedge set $\mathcal{E} \subseteq 2^{S}$, find a maximum size subset $\mathcal{E}^{\prime}$ of pairwise disjoint hyperedges. For our special case of Problem 1, this packing problem can be solved as follows: (a) for any natural number $k$ we can find a minimum cost subset $B_{k}$ of the arc set such that $B_{k}$ is the union of $k$ arc-disjoint $r$-arborescences, and (b) we can thus find (with a logarithmic search) the largest $k$ such that the cost of $B_{k}$ is $k$ times the cost of an optimal $r$-arborescence. Hypergraph packing and covering problems have an extensive literature, see for example the surveys [3] or [17, Part VIII.]. Most of these results deal with certain properties of hypergraphs (e.g. packing property, MFMC property, ideal hypergraphs, hypergraphs that pack, etc.) that help solving the packing and the covering problem. These results are LP based, as they use an LP relaxation of the packing/covering problem pair.

We looked at each of these general hypergraph covering frameworks in order to find a candidate to help solve Problems 1 and 2, to no avail. It seems that these problems are outside of the reach of the models known in the literature.

If the cost function $c$ is uniform then even the weighted Problem 2 is easy to solve (as the task is to find a minimum weight $r$-cut, where an $r$-cut is the set of arcs entering a non-empty subset $X \subseteq V-r)$. By a result of Edmonds [5] (see also [3, Theorem 1.24]) a polyhedral description can also be given. Namely the dominant of incidence vectors of $r$-cuts in a digraph $D=(V, A)$ can be described as $\left\{x \in \mathbb{R}^{A}: x \geq 0, x(B) \geq 1\right.$ for every $r$-arborescence $B\}$.

As we already mentioned in the introduction, Problems 1 and 2 are special cases of covering the minimum cost common bases of two matroids. It is not too hard to show, that given 2 matroids $M_{i}=\left(S, \mathcal{I}_{i}\right)(i=1,2)$ over the same ground set $S$ and a cost function $c: S \rightarrow \mathbb{R}$, the minimum cost common bases of $M_{1}$ and $M_{2}$ are 
the common bases of two other matroids $M_{1}^{\prime}$ and $M_{2}^{\prime}$ over the same ground set (see details in Section 6). That is, Problems 1 and 2 fall in the framework of covering the common bases of two matroids. Another natural special case of covering common bases is the following. Given a bipartite graph $G=(S, T, E)$, find a minimum size subset $H \subseteq E$ such that $G-H$ does not contain a perfect matching. However, this problem was shown to be NP-complete by Joret and Vetta [1].

Another related problem is the minimum cut problem in matroids. A cut of matroid is an inclusionwise minimal subset that intersects every base. The minimum cut problem in a matroid is the problem of finding a minimum size cut. This problem is also known as the cogirth problem (the cogirth of a matroid is the size of the smallest cut, while the girth of a matroid is the size of the smallest circuit). Since the cuts of a matroid are the circuits of the dual matroid, the minimum cut problem is equivalent with the minimum curcuit problem (a.k.a. girth problem). The (co)girth problem in matroids is NP-hard in general (e.g. girth is NP-hard in a transversal matroid [16], and both girth and cogirth are NP-hard in a binary matroid [18]). Polynomially solvable cases of the (co)girth problem has received considerable attention. For example, Geelen et al [9], motivated by applications in coding theory, devised a polynomial time randomized algorithm solving the (co)girth problem in binary matroids that arise from a graphic matroid by adding a small perturbation. The minimum cut problem is polynomially solvable in $k$-graphic-matroids (that is, matroids of the form $k M$ where $M$ is graphic); this was generalized by Király [13] for $k$-hypergraphic matroids (i.e. matroids of the form $k M$ where $M$ is hypergraphic; hypergraphic matroids were first defined by Lorea [15]).

Interdiction problems are also related to our research. In interdiction problems there are two (unrelated) objective functions like in Problem 2 (lets call them cost and weight), and we are interested in minimum cost objects satisfying some requirement (for example spanning trees, matchings, or shortest paths). The problem is then to increase the cost of these optimal objects to the greatest extent by removing elements with some budget for the total weight of the removed elements. We mention that there are other types of interdiction problems, too, but these are closest to our research. An example is the $k$ most vital edges problem: given an undirected graph with costs on the edges, and the task is to delete $k$ edges so that the minimum cost of a spanning tree in the remaining graph is as large as possible (in this case the weights are all 1, and $k$ plays the role of the budget). In [7] it is shown that this problem is NP-complete, and in [14] it is shown that it can be solved in time $O\left(n^{k+1}\right)$. The matching interdiction problem is the following. Given a graph $G$ with non-negative costs and weights on its edges and a budget, find a subset of edges $R$ such that the total cost of $R$ does not exceed the budget and the maximum weight of a matching in $G-R$ is smallest possible (note that for this sole problem we switched the role of the cost and the weight functions for conventional reasons). In [20, Theorem 3.3] it is shown that this problem is NP-complete on simple bipartite graphs with unit edge weights and unit interdiction costs, that is, it is NP hard to find a minimum size set of edges $F$ of a bipartite graph $G$ such that $F$ intersects every maximum matching of $G$ (as mentioned above, in [11] it is shown that this problem is NP-hard even for bipartite graphs that admit a perfect matching). In [19] a 4-approximation algorithm is given for the special case when all edge weights are 1. This is improved in [4] where it is shown that there exists a constant factor approximation algorithm for the matching interdiction problem even without this restriction on the edge weights.

\section{The problem and its variants}

In this paper we investigate Problem 1 and the more general Problem 2. For sake of simplicity we will mostly speak about Problem 1, and in Section 5.4 we sketch the necessary modifications of our algorithm needed to solve Problem 2. Note that Problem 2 with an integer weight function $w$ can be reduced to Problem 1 by replacing an arc $a \in A$ (of weight $w(a)$ ) with $w(a)$ parallel copies (each of weight 1 ). This reduction is however not polynomial. On the other hand, the algorithm we give for Problem 1 can be simply modified to solve Problem 2 in strongly polynomial time.

Let us give some more definitions. The arc set of the digraph $D$ will also be denoted by $A(D)$. Given a digraph $D=(V, A)$ and a node set $Z \subseteq V$, let $D[Z]$ be the digraph obtained from $D$ by deleting the nodes of $V-Z$ (and all the arcs incident with them). If $B \subseteq A$ is a subset of the arc set, then we will sometimes abuse the notation by identifying $B$ and the graph $(V, B)$ : thus $B[Z]$ is obtained from $(V, B)$ by deleting the nodes of $V-Z$ (and the arcs of $B$ incident with them). The set of $\operatorname{arcs}$ of $D$ entering (leaving) $Z$ is denoted $\delta_{D}^{i n}(Z)$ $\left(\delta_{D}^{\text {out }}(Z)\right)$, the number of these $\operatorname{arcs}$ is $\varrho_{D}(Z)=\left|\delta_{D}^{\text {in }}(Z)\right|\left(\delta_{D}(Z)=\left|\delta_{D}^{\text {out }}(Z)\right|\right.$, respectively). We will omit $D$ from the subscript in these notations, if no confusion can arise.

The following theorem of Fulkerson characterizes the minimum cost arborescences and leads us to a more convenient, but equivalent problem.

Theorem 1 (Fulkerson, [8]). There exists a subset $A^{\prime} \subseteq A$ of arcs (called tight arcs) and a laminar family $\mathcal{L} \subseteq 2^{V-r}$ such that an $r$-arborescence is of minimum cost if and only if it uses only tight arcs and it enters every member of $\mathcal{L}$ exactly once. The set $A^{\prime}$ and the family $\mathcal{L}$ can be found in polynomial time. 
Since non-tight arcs do not play a role in our problems, we can forget about them, so we assume that $A^{\prime}=A$ from now on.

Let $\mathcal{L}$ be a laminar family of subsets of $V$. A spanning arborescence $B \subseteq A$ in $D$ is called a $\mathcal{L}$-tight arborescence if both of the following hold.

1. $\left|\delta_{B}^{i n}(F)\right| \leq 1$ for all $F \in \mathcal{L}$, and

2. $\left|\delta_{B}^{i n}(F)\right|=0$ for all $F \in \mathcal{L}$ containing the root $r$ of $B$.

We point out that the second condition in the above definition is needed because we don't want to fix the root of the arborescences: this will be natural in the solution we give for Problem 1. The following statement gives an equivalent definition of $\mathcal{L}$-tight arborescences; the proof is left to the reader.

Claim 1. A spanning arborescence $B \subseteq A$ in $D$ is $\mathcal{L}$-tight if and only if $B[F]$ is an arborescence for every $F \in \mathcal{L}$.

The result of Fulkerson leads us to the following problem.

Problem 3. Given a digraph $D=(V, A)$, a designated root node $r \in V$ and a laminar family $\mathcal{L} \subseteq 2^{V}$, find a subset $H$ of the arc set such that $H$ intersects every $r$-rooted $\mathcal{L}$-tight arborescence and $|H|$ is minimum.

Note that in this problem we allow that $r \in F$ for some members $F \in \mathcal{L}$. By Fulkerson's Theorem above, if we have a polynomial algorithm for Problem 3 then we can also solve Problem 1 in polynomial time with this algorithm. However, this can be reversed by the next claim.

Claim 2. If we have a polynomial algorithm solving Problem 1 then we can also solve Problem 3 in polynomial time.

Proof. Let the cost of an arc $a \in A$ be equal to the number of sets $F \in \mathcal{L}$ such that $a$ enters $F$. Then an $r$-arborescence is of minimum cost if and only if it is $\mathcal{L}$-tight, if there exists an $\mathcal{L}$-tight arborescence at all.

We point out that the construction in the above proof also shows how to find an $\mathcal{L}$-tight arborescence, if it exists at all. So we can turn our attention to Problem 3. However, in order to have a more compact answer, it is more convenient to consider the following, equivalent problem instead, in which the root is not designated.

Problem 4. Given a digraph $D=(V, A)$ and a laminar family $\mathcal{L} \subseteq 2^{V}$, find a subset $H$ of the arc set such that $H$ intersects every $\mathcal{L}$-tight arborescence and $|H|$ is minimum.

Claim 3. There exists a polynomial algorithm solving Problem 3 if and only if there exists a polynomial algorithm solving Problem 4 .

Proof. Assume that there exists a polynomial time algorithm for Problem 4 and consider an instance of Problem 3. Since arcs entering $r$ will not be used in an optimal solution of Problem 3, we can assume that there are no such arcs. But then the $\mathcal{L}$-tight arborescences are all rooted in $r$, so our algorithm covering all $\mathcal{L}$-tight arborescences can be used for solving Problem 3 too.

For the other direction assume that we have a polynomial time algorithm solving Problem 3 and consider an instance of Problem 4 given with $D=(V, A)$ and $\mathcal{L} \subseteq 2^{V}$. Let $V^{\prime}=V+r^{\prime}$ with a new node $r^{\prime}$ and let $D^{\prime}=\left(V^{\prime}, A^{\prime}\right)$ where $A^{\prime}$ consists of the arcs in $A$ and an arc of multiplicity $|A|+1$ from $r^{\prime}$ to every $v \in V$. Finally let $\mathcal{L}^{\prime}=\mathcal{L}+\{V\}$. Now consider Problem 3 with input $D^{\prime}, r^{\prime}$ and $\mathcal{L}^{\prime}$. Observe that $\mathcal{L}$-tight arborescences in $D$ and $\left(r^{\prime}\right.$-rooted) $\mathcal{L}^{\prime}$-tight arborescences in $D^{\prime}$ correspond to each-other in a natural way, and an optimal solution to this instance of Problem 3 will not contain any arc of form $r^{\prime} v$ (if it contains one then it has to contain all parallel copies, but we included those with a large multiplicity).

The main result of this paper is a polynomial algorithm solving Problem 4 , and thus, by Claims 2 and 3 , for Problems 1 and 3. For a digraph $D=(V, A)$, and a laminar family $\mathcal{L}$ of subsets of $V$, let $\gamma(D, \mathcal{L})$ denote the minimum number of arcs deleted from $D$ to obtain a digraph that does not contain an $\mathcal{L}$-tight arborescence, that is,

$$
\gamma(D, \mathcal{L}):=\min \{|H|: H \subseteq A \text { such that } D-H \text { contains no } \mathcal{L} \text {-tight arborescence }\} \text {. }
$$

Notice that an arborescence is $\mathcal{L}$-tight if and only if it is $(\mathcal{L} \cup\{V\})$-tight, and $\gamma(D, \mathcal{L})=\gamma(D, \mathcal{L} \cup\{V\})$. Therefore we will assume that $V \in \mathcal{L}$ from now on. 


\section{Covering all arborescences - a special case}

In the proof of our main result below, we will use its special case when the laminar family $\mathcal{L}=\{V\}$. This special case amounts to the following well-known characterization of the existence of a spanning arborescence.

Lemma 1. For any digraph $D=(V, A)$ exactly one of the following two alternatives holds:

1. there exists a spanning arborescence,

2. there exist two disjoint non-empty subsets $Z_{1}, Z_{2} \subset V$ such that $\varrho_{D}\left(Z_{1}\right)=\varrho_{D}\left(Z_{2}\right)=0$.

This characterization also implies a formula to determine the minimum number of edges to be deleted to destroy all arborescences. The characterization is based on double cuts.

Definition 1. For a digraph $D=(V, A)$, a double cut $\delta^{i n}\left(Z_{1}\right) \cup \delta^{i n}\left(Z_{2}\right)$ is determined by a pair of non-empty disjoint node subsets $Z_{1}, Z_{2} \subseteq V$. The minimum cardinality of a double cut is denoted by $\mu(D)$, that is

$$
\mu(D):=\min \left\{\left|\delta^{i n}\left(Z_{1}\right)\right|+\left|\delta^{i n}\left(Z_{2}\right)\right|: Z_{1} \cap Z_{2}=\emptyset \neq Z_{1}, Z_{2} \subsetneq V\right\} .
$$

Corollary 1. For any digraph $D=(V, A)$ the following equation holds: $\gamma(D, \emptyset)=\mu(D)$.

We point out that a minimum double cut can be found in polynomial time by a simple reduction to minimum cut: this is described in Section 5.5. Furthermore we will need the following observation (the proof is left to the reader).

Lemma 2. Given a digraph $D=(V, A)$, let $R=\{r \in V$ : there exists an $r$-rooted spanning arborescence in $D\}$. Then $D[R]$ is a strongly connected digraph, and $\varrho_{D}(R)=0$.

\section{Covering tight arborescences}

Given a laminar family $\mathcal{L} \subseteq 2^{V}$ with $V \in \mathcal{L}$, for $F \in \mathcal{L}$, let $\mathcal{L}[F]:=\left\{F^{\prime} \in \mathcal{L}, F^{\prime} \subseteq F\right\}$. A simple corollary of Claim 1 is the following.

Claim 4. For any $\mathcal{L}$-tight arborescence $B$, and any $F \in \mathcal{L}, B[F]$ is an $\mathcal{L}[F]$-tight arborescence in $D[F]$.

The following observation is crucial in our proofs. Given a digraph $D=(V, A)$ and a laminar family $\mathcal{L} \subseteq 2^{V}$, for an arbitrary member $F \in \mathcal{L}$ and $\operatorname{arc} a=x y \in A$ leaving $F$, let $\tilde{D}$ be the graph obtained from $D$ by changing the tail of $a$ for an arbitrary other node $x^{\prime} \in F$, that is $\tilde{D}=D-x y+x^{\prime} y$ (where $x, x^{\prime} \in F$ and $y \notin F$ ). This operation will be called a tail-relocation. Clearly, there is a natural bijection between the $\operatorname{arcs}$ of $D$ and those of $\tilde{D}$, but even more importantly, this bijection also induces a bijection between the $\mathcal{L}$-tight arborescences in $D$ and those in $\tilde{D}$. This is formulated in the following claim.

Claim 5. Let $B \subseteq A$ and $x y \in B$. Then $B-x y+x^{\prime} y$ is an $\mathcal{L}$-tight arborescence in $\tilde{D}$ if and only if $B$ is an $\mathcal{L}$-tight arborescence in $D$.

The claim also implies that $\gamma(D, \mathcal{L})=\gamma(\tilde{D}, \mathcal{L})$.

\subsection{A "min-min" formula}

Our approach to determine $\gamma(D, \mathcal{L})$ is broken down into two steps. First, we prove a "min-min" formula, that is, we show that a set $H$ that attains the minimum in (1) is equal to a special arc subset called an $\mathcal{L}$-double-cut. The second step will be the construction of an algorithm to find a minimum cardinality $\mathcal{L}$-double-cut.

So what is this first step - the min-min formula all about? It expresses that in order to cover optimally the $\mathcal{L}$-tight arborescences we need to consider the problem of covering the $\mathcal{L}[F]$-tight arborescences for every $F \in \mathcal{L}$.

Definition 2. For a set $Z \subseteq V$, let $\mathcal{L}_{Z}$ denote the family of sets in $\mathcal{L}$ not disjoint from $Z$, that is, let

$$
\mathcal{L}_{Z}:=\{F \in \mathcal{L}: F \cap Z \neq \emptyset\} .
$$

Then an $\mathcal{L}$-cut $M(Z)$ is defined as the set of arcs entering $Z$, but not leaving any set in $\mathcal{L}_{Z}$, that is, let

$$
M(Z):=M_{D, \mathcal{L}}(Z):=\delta_{D}^{\text {in }}(Z)-\bigcup_{F \in \mathcal{L}_{Z}}\left(\delta_{D}^{\text {out }}(F)\right) .
$$




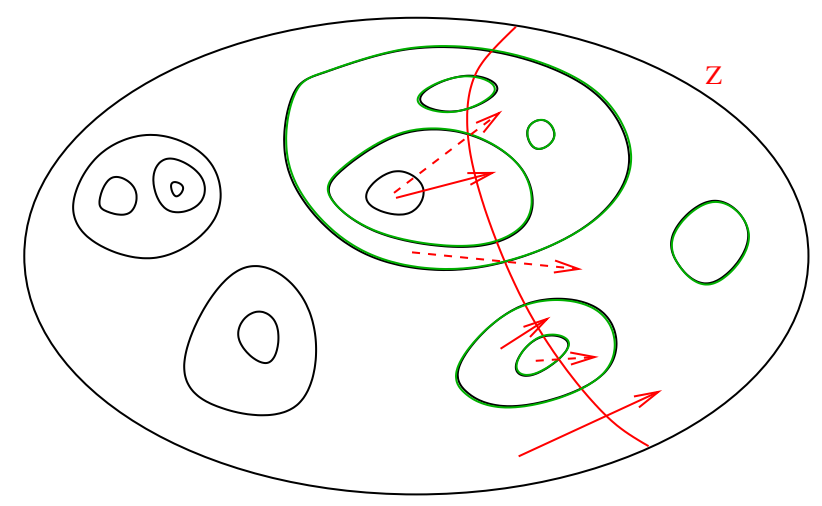

Figure 1: Illustration of an $\mathcal{L}$-cut. The arcs drawn with a solid line count in $M(Z)$, those with dashed line do not.

Thus $M(Z)$ consists of those arcs entering $Z$, but not leaving any of those sets in $\mathcal{L}$ that have non-empty intersection with $Z$. An illustration can be found in Figure 1 . A set function $f$ is given by the cardinality of an $\mathcal{L}$-cut, that is, we define

$$
f(Z):=f_{D}(Z):=f_{D, \mathcal{L}}(Z):=\left|M_{D, \mathcal{L}}(Z)\right|
$$

It is useful to observe that

$$
f_{D, \mathcal{L}}(Z) \geq f_{D[F], \mathcal{L}[F]}(Z \cap F) \text { for any } F \in \mathcal{L} .
$$

The motivation for $f$ and $M(Z)$ is that $H=M(Z)$ is a set of arcs the deletion of which destroys all tight arborescences rooted outside of $Z$, as claimed by the following lemma.

Lemma 3. Given $D=(V, A)$ and laminar family $\mathcal{L} \subseteq 2^{V}$. If $f_{D}(Z)=0$ for some non-empty $Z \subseteq V$ then there is no $\mathcal{L}$-tight arborescence in $D$ with root outside $Z$.

Proof. Take an arbitrary arc $u v$ entering $Z$. Since $u v \notin M(Z)$, there exists an $u \bar{v}$ set $F \in \mathcal{L}$ so that $F \cap Z \neq \emptyset$. Relocate the tail of this arc into a node in $F \cap Z$. Let $D^{\prime}$ be the digraph obtained from $D$ after doing this tail-relocation for every arc entering $Z$. By Claim 5 , there exists an $\mathcal{L}$-tight arborescence in $D$ with root outside $Z$ if and only if there exists an $\mathcal{L}$-tight arborescence in $D^{\prime}$ with root outside $Z$. But $\varrho_{D^{\prime}}(Z)=0$.

For any $F \in \mathcal{L}$ and nonempty disjoint subsets $Z_{1}, Z_{2} \subseteq F$ the set of $\operatorname{arcs}$ in $M_{D[F], \mathcal{L}[F]}\left(Z_{1}\right) \cup M_{D[F], \mathcal{L}[F]}\left(Z_{2}\right)$ will be called an $\mathcal{L}$-double cut, and we introduce the following notation for the minimum cardinality of an $\mathcal{L}$-double cut:

$$
\Theta_{F}:=\Theta_{F, D}:=\Theta_{F, D, \mathcal{L}}:=\min \left\{f_{D[F], \mathcal{L}[F]}\left(Z_{1}\right)+f_{D[F], \mathcal{L}[F]}\left(Z_{2}\right): \emptyset \neq Z_{1}, Z_{2} \subseteq F, Z_{1} \cap Z_{2}=\emptyset\right\} .
$$

The following simple observation is worth mentioning.

Claim 6. Given a digraph $D=(V, A)$ and a laminar family $\mathcal{L} \subseteq 2^{V}$, then $f_{D, \mathcal{L}}(Z) \leq \varrho_{D}(Z)$ holds for every $Z \subseteq V$. Consequently, $\Theta_{F, D, \mathcal{L}} \leq \mu(D[F])$ holds for any $F \in \mathcal{L}$.

Note that the tail-relocation operation introduced above does not change the $f$-value of any set $Z \subseteq V$, that is $f_{D, \mathcal{L}}(Z)=f_{D^{\prime}, \mathcal{L}}(Z)$, if $D^{\prime}$ is obtained from $D$ by (one or several) tail-relocation. Consequently, this operation does not modify the $\Theta$ value, either, that is $\Theta_{F, D, \mathcal{L}}=\Theta_{F, D^{\prime}, \mathcal{L}}$ for any $F \in \mathcal{L}$. The following "min-min" theorem motivates the definition of $\Theta$.

Theorem 2. For a digraph $D=(V, A)$, and a laminar family $\mathcal{L}$ of subsets of $V$, the minimum number of arcs to be deleted from $D$ to obtain a digraph that does not contain an $\mathcal{L}$-tight arborescence is attained on an $\mathcal{L}$-double cut, that is

$$
\gamma(D, \mathcal{L})=\min _{F \in \mathcal{L}} \Theta_{F, D, \mathcal{L}}
$$

Proof. By Lemma 3, $\gamma(D, \mathcal{L}) \leq \min _{F \in \mathcal{L}} \Theta_{F}$, since if we delete an arc set $M_{D[F]}\left(Z_{1}\right) \cup M_{D[F]}\left(Z_{2}\right)$ for some $F \in \mathcal{L}$ and non-empty disjoint $\bar{Z}_{1}, Z_{2} \subseteq F$, then no $\mathcal{L}[F]$-tight arborescence survives in $D[F]$ (since its root can neither be in $F-Z_{1}$, nor in $F-Z_{2}$, by Lemma 3 , and $\left.F=\left(F-Z_{1}\right) \cup\left(F-Z_{2}\right)\right)$.

The other inequality follows from Lemma 4 below.

Lemma 4. Given a digraph $D=(V, A)$ and a laminar family $\mathcal{L} \subseteq 2^{V}$ with $V \in \mathcal{L}$, if

$$
f_{D[F]}\left(Z_{1}\right)+f_{D[F]}\left(Z_{2}\right) \geq 1 \text { for any } F \in \mathcal{L} \text { and non-empty disjoint sets } Z_{1}, Z_{2} \subseteq F
$$

then there exists a $\mathcal{L}$-tight arborescence in $D$. 
Proof. Let $\bar{D}=(V+\bar{r}, A \cup\{\bar{r} v: v \in V\})$. Notice that $\mathcal{L}$-tight arborescences in $D$ and $\mathcal{L}$-tight arborescences in $\bar{D}$ naturally correspond to each other, since $V \in \mathcal{L}$. Thus we will show that there exists an $\mathcal{L}$-tight arborescence in $\bar{D}$ (notice that the condition holds for $\bar{D}$, since $\bar{D}[F]=D[F]$ for every $F \in \mathcal{L}$ ). We will use induction on $|\mathcal{L}|+|V|+|A(\bar{D})|$. If $\mathcal{L}=\{V\}$ then the lemma is true by Lemma 1. Otherwise let $F \in \mathcal{L}$ be an inclusionwise minimal member of $\mathcal{L}$ : again by Lemma 1 , there exists a spanning arborescence in $\bar{D}[F]$. Let $R$ be the subset of nodes of $F$ that can be the root of a spanning arborescence in $\bar{D}[F]$, i.e. $R=\{r \in F$ : there exists an $r$-rooted arborescence (spanning $F$ ) in $\bar{D}[F]\}$.

1. Assume first that $|R| \geq 2$ and let $\bar{D}_{1}=\bar{D} / R$ obtained by contracting $R$. For any set $Z \subseteq V$ which is either disjoint form $R$, or contains $R$, let $Z / R$ be its (well-defined) image after the contraction and let $\mathcal{L}_{1}=\{X / R: X \in \mathcal{L}\}$. By induction, there exists an $\mathcal{L}_{1}$-tight arborescence $P$ in $\bar{D}_{1}$, since $f_{\bar{D}[X / R]}(Z / R)=$ $f_{\bar{D}[X]}(Z)$ for any $X / R \in \mathcal{L}_{1}$ and $Z / R \subseteq X / R$. It is clear that we can create an $\mathcal{L}$-tight arborescence in $\bar{D}$ from $P$ : we describe one possible way. Consider the unique arc in $P$ that enters $F$ and assume that the pre-image of this arc has head $r \in R$. Delete every arc from $P$ induced by $F / R$ and substitute them with an arbitrary $r$-rooted arborescence (spanning $F$ ) of $\bar{D}[F]$. This clearly gives an $\mathcal{L}$-tight arborescence.

2. So we can assume that $R=\{r\}$. Next assume that there exists an arc $u v \in A(\bar{D})$ entering $F$ with $r \neq v$. Let $\bar{D}_{2}=\bar{D}-u v$ : we claim that there exists an $\mathcal{L}$-tight arborescence in $\bar{D}_{2}$ (which is clearly an $\mathcal{L}$-tight arborescence in $\bar{D}$, too). If this does not hold then by the induction there must exist a set $F^{\prime} \in \mathcal{L}$ and non-empty disjoint subsets $Z_{1}, Z_{2} \subseteq F^{\prime}$ with $\sum_{i=1,2} f_{\bar{D}_{2}\left[F^{\prime}\right]}\left(Z_{i}\right)=0$. Since $\sum_{i=1,2} f_{\bar{D}\left[F^{\prime}\right]}\left(Z_{i}\right)>0$, the arc $u v$ must be equal to (say) $M_{\bar{D}\left[F^{\prime}\right], \mathcal{L}}\left(Z_{1}\right)$ (while $M_{\bar{D}\left[F^{\prime}\right], \mathcal{L}}\left(Z_{2}\right)=\emptyset$ ). This implies that $u v$ enters $Z_{1}$, while $r \in Z_{1}$ must also hold, otherwise $f_{\bar{D}\left[F^{\prime}\right]}\left(Z_{1}\right) \geq 2$ would hold, since $v$ is reachable from $r$ in $\bar{D}\left[F^{\prime}\right]$. Let $Z_{1}^{\prime}=Z_{1}-(F-r)$ and observe that $f_{\bar{D}\left[F^{\prime}\right]}\left(Z_{1}^{\prime}\right)=0$ : this is because the arcs in $\delta_{\bar{D}\left[F^{\prime}\right]}^{i n}\left(Z_{1}^{\prime}\right)-\delta_{\bar{D}\left[F^{\prime}\right]}^{i n}\left(Z_{1}\right)$ all leave $F$, since $\varrho_{\bar{D}[F]}(r)=0$ by Lemma 2. Thus $f_{\bar{D}\left[F^{\prime}\right]}\left(Z_{1}^{\prime}\right)+f_{\bar{D}\left[F^{\prime}\right]}\left(Z_{2}\right)=0$, a contradiction.

3. Thus we can also assume that the arcs of $\bar{D}$ entering $F$ all enter $r$. Let $\mathcal{L}_{2}=\mathcal{L}-\{F\}$ : then clearly $f_{\bar{D}\left[F^{\prime}\right], \mathcal{L}_{2}}(Z) \geq f_{\bar{D}\left[F^{\prime}\right], \mathcal{L}}(Z)$ for any $F^{\prime} \in \mathcal{L}_{2}$ and $Z \subseteq F^{\prime}$, so by induction there exists an $\mathcal{L}_{2}$-tight arborescence $P_{2}$ in $\bar{D}$. Since the root of $P_{2}$ is $\bar{r}$ and $P_{2}$ enters $F$ only once (since all arcs entering $F$ have head $r), P_{2}$ is also $\mathcal{L}$-tight, so the theorem is proved.

\subsection{A second proof of the "min-min" theorem}

In this subsection we reprove Lemma 4 which is the hard direction of Theorem 2, Our motivation is that we think we can give better insights into the details of our techniques.

Let $\mathcal{L}_{0}=\{\{v\}: v \in V\} \cup\{V\}$. Notice that an arborescence is $\mathcal{L}$-tight if and only if it is $\left(\mathcal{L} \cup \mathcal{L}_{0}\right)$-tight $($ and $\mathcal{L} \cup \mathcal{L}_{0}$ is also laminar, if $\mathcal{L}$ is laminar). Therefore, in Section 5.2 we will assume that $\mathcal{L}_{0} \subseteq \mathcal{L}$. In what follows we abbreviate $f_{D}$ by $f$ (and $f_{D[F], \mathcal{L}[F]}$ is abbreviated by $f_{D[F]}$ for an $F \in \mathcal{L}$ ). For nodes $s, t \in V$, a subset $S$ with $s \in S \subseteq V-t$ is called an $s \bar{t}$-set.

Claim 7. Given a set $Z \subseteq V$, an arc st is in $M(Z)$ if and only if it enters $Z$ and the largest sit set in $\mathcal{L}$ is disjoint from $Z$.

Notice that the largest $s \bar{t}$ set in $\mathcal{L}$ is well defined for every pair $s, t \in V$, since $\mathcal{L}_{0} \subseteq \mathcal{L}$; in fact this motivates the introduction of $\mathcal{L}_{0}$.

Claim 8. For sets $X, Y \subseteq V$ we have $M(X \cup Y) \subseteq M(X) \cup M(Y)$. Consequently, $f(X)=f(Y)=0$ implies $f(X \cup Y)=0$.

Proof. Consider an arc $a \in M(X \cup Y)$ : this must enter at least one of $X$ and $Y$, say it enters $X$. We claim that $a \in M(X)$, too: it cannot leave some $F \in \mathcal{L}_{X}$, since $\mathcal{L}_{X} \subseteq \mathcal{L}_{X \cup Y}$.

Claim 9. If (7) holds and $f(X)=f(Y)=0$ for some $X, Y \subseteq V$ then $f(X \cap Y)=0$, too. (Note that this does not necessarily hold without (7).)

Proof. Assume $f(X \cap Y)>0$ and consider an arc $u v \in M(X \cap Y)$. Let $F \in \mathcal{L}$ be the largest $u \bar{v}$ set in $\mathcal{L}$. Since $u v \in M(X \cap Y), F \cap(X \cap Y)=\emptyset$, but since $f(X)=f(Y)=0, F \cap X \neq \emptyset \neq F \cap Y$, that together with $f_{D[F]}(X \cap F)=f_{D[F]}(Y \cap F)=0$ contradicts $[7]$.

The following statement extends parts of Lemma 2

Claim 10. Given a digraph $D=(V, A)$ and laminar family $\mathcal{L} \subseteq 2^{V}$, let $R=\{r \in V$ : there exists a $\mathcal{L}$-tight arborescence in $D$ with root $r\}$. Then $f(R)=0$. 
Proof. Assume $f(R)>0$ and let $u v \in M(R)$. Let $F$ be the largest $u \bar{v}$ set in $\mathcal{L}$. We have $F \cap R=\emptyset$. Let $B \subseteq A$ be an $\mathcal{L}$-tight arborescence of root $v$, and let $x y \in B$ be the (unique) arc entering $F$. Consider $B^{\prime}=B+u v-x y$ : this is an arborescence of root $y$, since every node has in-degree 1 , and $B^{\prime}$ is a spanning tree in the undirected sense. We claim that $B^{\prime}$ is also $\mathcal{L}$-tight which contradicts the definition of $R$.

Proof of Lemma 4. The proof is by induction on $|\mathcal{L}|+|A|$. The base case $\mathcal{L}=\mathcal{L}_{0}$ is clear. Assume $\mathcal{L} \supsetneq \mathcal{L}_{0}$ and let $F_{0} \in \mathcal{L}$ be an inclusionwise maximal member of $\mathcal{L}-\mathcal{L}_{0}$. An arc in $u v \in \delta^{\text {in }}\left(F_{0}\right)$ is called erasable if (7) holds for $\mathcal{L}$ and $D-u v$, otherwise $u v$ is called essential. If there exists an erasable arc then we are done by induction. So we can assume that every arc entering $F_{0}$ is essential. We will prove that the number of arcs entering $F_{0}$ is at most one. In order to prove this, let us first investigate when an arc is essential. Let $R=\left\{r \in F_{0}: r\right.$ can be the root of an $\mathcal{L}\left[F_{0}\right]$-tight arborescence in $\left.D\left[F_{0}\right]\right\}$. By the induction hypothesis, $R \neq \emptyset$. An $\operatorname{arc} u v$ entering $F_{0}$ is essential if and only if there exist non-empty disjoint $Z_{1}, Z_{2} \subseteq V$ with $M\left(Z_{1}\right)=\{u v\}$ and $M\left(Z_{2}\right)=\emptyset$ (here we used that $F_{0}$ is a maximal member of $\mathcal{L}$ ): assume that this is the case. Observe that $f_{D\left[F_{0}\right]}\left(Z_{1} \cap F_{0}\right)=0$, implying that

1. $F_{0} \cap Z_{2}=\emptyset$, otherwise $f_{D\left[F_{0}\right]}\left(Z_{1} \cap F_{0}\right)=f_{D\left[F_{0}\right]}\left(Z_{2} \cap F_{0}\right)=0$ would contradict (7), and

2. $R \subseteq Z_{1}$, by the definition of $R$ and Lemma 3 .

Next we prove that $f\left(Z_{1}-\left(F_{0}-R\right)\right) \leq f\left(Z_{1}\right)$. Let $\hat{Z}_{1}=Z_{1}-\left(F_{0}-R\right)$ : in fact we show $M\left(\hat{Z}_{1}\right) \subseteq M\left(Z_{1}\right)$. Consider an arc $s t \in M\left(\hat{Z}_{1}\right)$. If $s \notin F_{0}$ then the largest $s \bar{t}$ set in $\mathcal{L}$ must be disjoint from $F_{0}$, therefore $s t \in M\left(Z_{1}\right)$, too. So assume $s \in F_{0}$. Then $t$ cannot be in $F_{0}$ (that is, in $R$ ), since that would imply $f_{D\left[F_{0}\right]}(R)>0$, contradicting Claim 10. Therefore $t \notin F_{0}$, that is, $F_{0}$ is an $s \bar{t}$ member of $\mathcal{L}$, contradicting $s t \in M\left(\hat{Z}_{1}\right)$ by Claim 7 (note that $F_{0} \cap Z_{1}$ equals the nonempty set $R$ ).

The previous observation implies that $v \in R$ must hold (otherwise $f\left(\hat{Z}_{1}\right)+f\left(Z_{2}\right)=0$ ). So we can assume that $Z_{1} \cap F_{0}=R$ (we can substitute $Z_{1}$ by $\hat{Z}_{1}$ ).

Assume that there is another arc $u^{\prime} v^{\prime}$ entering $F_{0}$. By our assumption, this arc is essential, proven by the existence of non-empty disjoint $Z_{1}^{\prime}, Z_{2}^{\prime} \subseteq V$ with $M\left(Z_{1}^{\prime}\right)=\left\{u^{\prime} v^{\prime}\right\}$ and $M\left(Z_{2}^{\prime}\right)=\emptyset$. Notice that $Z_{2} \cap Z_{2}^{\prime} \neq \emptyset$ by (7). We can again assume that $Z_{1}^{\prime} \cap F_{0}=R$ and $v^{\prime} \in R$. By Claim $8, M\left(Z_{1} \cup Z_{1}^{\prime}\right) \subseteq\left\{u v, u^{\prime} v^{\prime}\right\}$. However, $M\left(Z_{1} \cup Z_{1}^{\prime}\right)=\emptyset$, for example $u v \notin M\left(Z_{1} \cup Z_{1}^{\prime}\right)$, since $u v$ enters $Z_{1} \cap Z_{1}^{\prime}$ and $u v \notin M\left(Z_{1}^{\prime}\right)$, either $u v$ does not enter $Z_{1}^{\prime}$, or if it enters, then the largest $u \bar{v}$ set in $\mathcal{L}$ intersects $Z_{1}^{\prime}$. We have a contradiction with (7) having $f\left(Z_{1} \cup Z_{1}^{\prime}\right)=f\left(Z_{2} \cap Z_{2}^{\prime}\right)=0$.

We have proved that $\varrho_{D}\left(F_{0}\right) \leq 1$. Let $\mathcal{L}^{\prime}=\mathcal{L}-\left\{F_{0}\right\}$. The conditions in (7) clearly hold for $\mathcal{L}^{\prime}$ (since $f_{D[F], \mathcal{L}^{\prime}}(Z) \geq f_{D[F], \mathcal{L}}(Z)$ for every $F \in \mathcal{L}^{\prime}$ and $\left.Z \subseteq F\right)$, so by induction there exists an $\mathcal{L}^{\prime}$-tight arborescence $B \subseteq A$. If $\varrho_{D}\left(F_{0}\right)=0$ then $B$ is also $\mathcal{L}$-tight. On the other hand, if $u v \in A$ enters $F_{0}$, then $u v$ was essential (proven by non-empty disjoint $Z_{1}, Z_{2} \subseteq V$ with $M\left(Z_{1}\right)=\{u v\}$ and $M\left(Z_{2}\right)=\emptyset$ ), but then $Z_{2} \cap F_{0}=\emptyset$, as we observed earlier, implying (by Lemma 3 that the root of $B$ can not be in $F_{0}$, so $B$ is also $\mathcal{L}$-tight, what was to be proven.

\subsection{In-solid sets and anchor nodes}

Theorem 2 suggests a strategy for solving Problem 1 ; for every $F \in \mathcal{L}$ we find a minimum $\mathcal{L}[F]$-double-cut in $D[F]$ and output the best of these. For finding the minimum $\mathcal{L}[F]$-double-cut, tail relocation can help: if $\Theta_{F}=f_{D[F], \mathcal{L}[F]}\left(Z_{1}\right)+f_{D[F], \mathcal{L}[F]}\left(Z_{2}\right)$ for some nonempty, disjoint pair $Z_{1}, Z_{2} \subseteq F$, then suitably relocating the tails of all arcs that leave some $F^{\prime} \in \mathcal{L}[F]_{Z_{i}}$ to a node in $F^{\prime} \cap Z_{i}$ we do not change $\Theta_{F}$ and $f_{D[F]}\left(Z_{i}\right)$ becomes $\varrho_{D^{\prime}[F]}\left(Z_{i}\right)$, thus the problem boils down to finding a minimum double cut in $D^{\prime}[F]$ (where $D^{\prime}$ is obtained from $D$ after some suitable tail-relocations). But we can not try every possible tail-relocations, there are too many of those, we need something more here. These extra ideas are introduced in this section.

Definition 3. A family of sets $\mathcal{F} \subseteq 2^{V}$ of a finite ground set $V$ is said to satisfy the Helly-property, if any sub-family $\mathcal{X}$ of pairwise intersecting members of $\mathcal{F}$ has a non-empty intersection, i.e. $\mathcal{X} \subseteq \mathcal{F}$ and $X \cap X^{\prime} \neq \emptyset$ for every $X, X^{\prime} \in \mathcal{X}$ implies that $\cap \mathcal{X} \neq \emptyset$.

The following definition is taken from [1].

Definition 4. Given a digraph $G=(V, A)$, a non-empty subset of nodes $X \subseteq V$ is called in-solid, if $\varrho(Y)>\varrho(X)$ holds for every nonempty $Y \subsetneq X$.

Theorem 3 (Bárász, Becker, Frank [1]). The family of in-solid sets of a digraph satisfies the Helly-property.

The authors of [1] prove in fact more: they show that the family of in-solid sets is a subtree-hypergraph, but we will only use the Helly property here. The following theorem formulates the key observation for the main result. 
Theorem 4. In a digraph $G=(V, A)$ there exists a node $t \in V$ such that $\varrho(Z) \geq \frac{\mu(G)}{2}$ for every non-empty $Z \subseteq V-t$.

Proof. Consider the family $\mathcal{X}=\left\{X \subseteq V: X\right.$ is in-solid and $\left.\varrho(X)<\frac{\mu(G)}{2}\right\}$. If there were two disjoint members $X, X^{\prime} \in \mathcal{X}$ then $\varrho(X)+\varrho\left(X^{\prime}\right)<\mu(G)$ would a contradict the definition of $\mu(G)$. Therefore, by the Hellyproperty of the in-solid sets, there exists a node $t \in \cap \mathcal{X}$. This node satisfies the requirements of the theorem, since if there was a non-empty $Z \subseteq V-t$ with $\varrho(Z)<\frac{\mu(G)}{2}$, then $Z$ would necessarily contain an in-solid set $Z^{\prime} \subseteq Z$ with $\varrho\left(Z^{\prime}\right) \leq \varrho(Z)$ (this follows from the definition of in-solid sets), contradicting the choice of $t$.

In a digraph $G=(V, A)$, a node $a \in V$ with the property $\varrho(Z) \geq \frac{\mu(G)}{2}$ for every non-empty $Z \subseteq V-a$ will be called an anchor node of $G$.

\subsection{A polynomial-time algorithm}

In this section we present a polynomial time algorithm to determine the robustness of tight arborescences, which also implies a polynomial time algorithm to determine the robustness of minimum cost arborescences. A sketch of the algorithm goes as follows. We maintain a subset $\mathcal{L}^{\prime}$ of $\mathcal{L}$, which is initiated with $\mathcal{L}^{\prime}:=\mathcal{L}$. For a minimal member $F$ of $\mathcal{L}^{\prime}$, we apply Theorem 4 , and find an anchor node $a_{F}$ of $D[F]$. We replace the tail of every arc leaving $F$ by $a_{F}$, remove $F$ from $\mathcal{L}^{\prime}$, and repeat until $\mathcal{L}^{\prime}$ goes empty. This way we construct a sequence of digraphs on the same node set: let $D^{\prime}$ be the last member of this sequence. Then for any $a \in V$ we construct another digraph $D_{a}$ from $D^{\prime}$ : for every $F \in \mathcal{L}$ with $a \in F$ and every arc of $D^{\prime}$ leaving $F$ we replace the tail of this arc with $a$. Finally, we determine minimum double cuts in $D^{\prime}[F]$ for every $F \in \mathcal{L}$, and we also determine minimum double cuts in $D_{a}[F]$ for every $F \in \mathcal{L}$ with $a \in F$ : this way we have determined $O\left(n^{2}\right)$ double cuts altogether. Each of these double cuts also determines an $\mathcal{L}$-double cut in $D$, and we pick the one with the smallest cardinality, to claim that it actually is optimal. The algorithm is given as a pseudocode below.

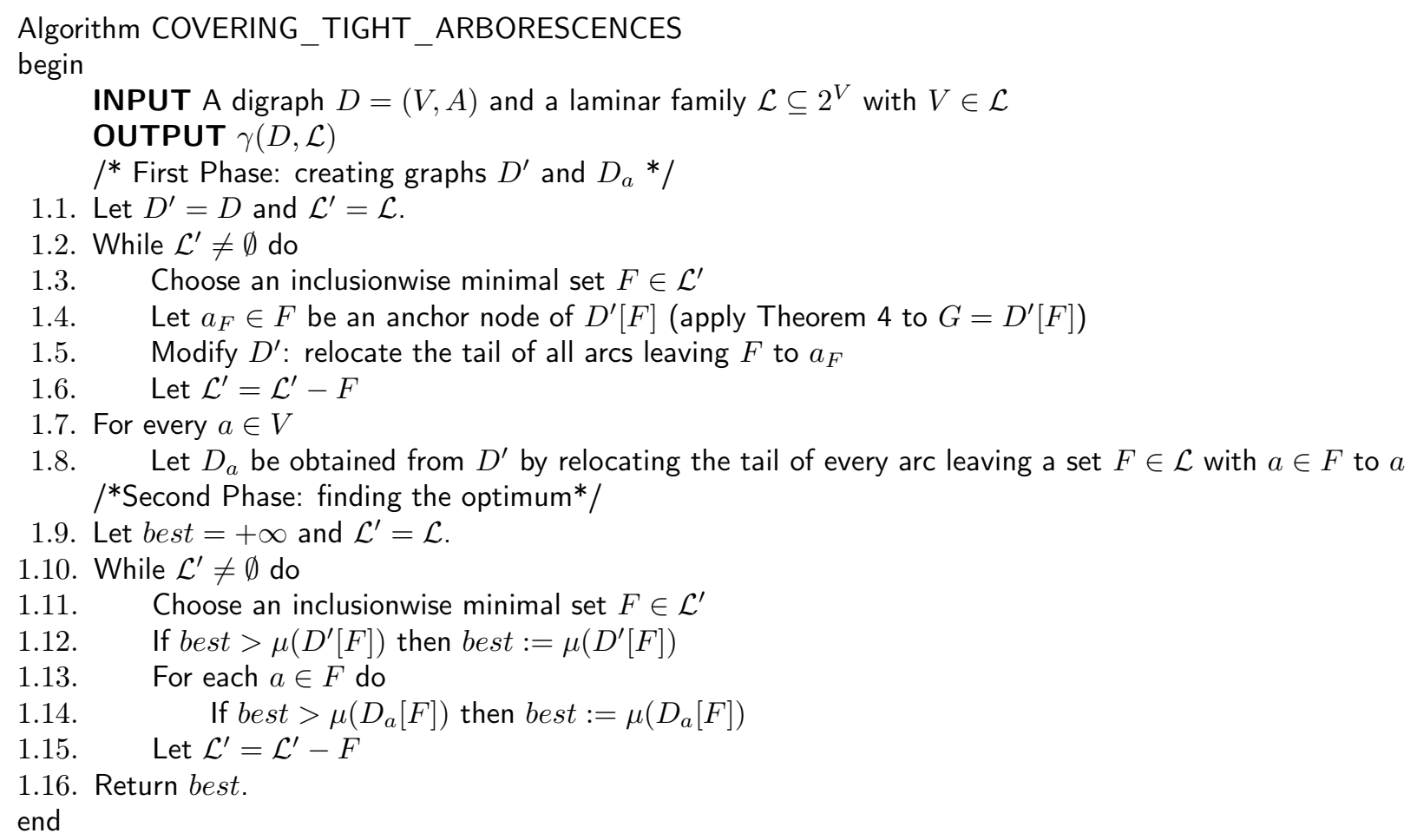

The algorithm above is formulated in a way that it returns the optimum $\gamma(D, \mathcal{L})$ in question, but by the correspondance between the arc set of $D$ and that of $D^{\prime}$ and $D_{a}$ in the algorithm, clearly we can also return the optimal arc set, too. It is also clear that the algorithm can be formulated to run in strongly polynomial time for Problem 2, too: we only need to modify the definition of the in-degree function $\varrho_{D}$, so that the weights are taken into account.

Theorem 5. The Algorithm COVERING_TIGHT_ARBORESCENCES returns a correct answer.

Proof. First of all, since $\Theta_{F, D}=\Theta_{F, D^{\prime}}=\Theta_{F, D_{a}}$ for any $F \in \mathcal{L}$ and $a \in F$, and $\Theta_{F, D^{\prime}} \leq \mu\left(D^{\prime}[F]\right)$ and $\Theta_{F, D_{a}} \leq \mu\left(D_{a}[F]\right)$, the algorithm returns an upper bound for the optimum $\gamma(D, \mathcal{L})$ in question by Theorem 2

On the other hand, assume that $F$ is an inclusionwise minimal member of $\mathcal{L}$ such that the optimum $\gamma(D, \mathcal{L})=$ $\Theta_{F, D}$ (such a set exists again by Theorem 2). Assume furthermore that the non-empty disjoint sets $Z_{1}, Z_{2} \subseteq F$ are such that $\Theta_{F, D}=f_{D[F]}\left(Z_{1}\right)+f_{D[F]}\left(Z_{2}\right)$. The following sequence of observations proves the theorem. 
1. First observe, that any member $F^{\prime} \in \mathcal{L}$ which is a proper subset of $F$ can intersect at most one of $Z_{1}$ and $Z_{2}$. Assume the contrary, and note that $f_{D[F]}\left(Z_{i}\right) \geq f_{D\left[F^{\prime}\right]}\left(Z_{i} \cap F^{\prime}\right)$ holds for $i=1,2$, contradicting the minimal choice of $F$.

2. Next observe that there do not exist two disjoint members $F^{\prime}, F^{\prime \prime} \in \mathcal{L}_{Z_{1} \cup Z_{2}}$ that are proper subsets of $F$ such that $a_{F^{\prime}}$ and $a_{F^{\prime \prime}}$ are both outside $Z_{1} \cup Z_{2}$. To see this assume again the contrary and let $F^{\prime}, F^{\prime \prime}$ be two inclusionwise minimal such sets. By exchanging the roles of $Z_{1}$ and $Z_{2}$ or the roles of $F^{\prime}$ and $F^{\prime \prime}$ we arrive at the following two cases: either both $F^{\prime}$ and $F^{\prime \prime}$ intersect $Z_{1}$, or $F^{\prime}$ intersects $Z_{1}$ and $F^{\prime \prime}$ intersects $Z_{2}$. The proof is analogous for both cases. Assume first that both $F^{\prime}$ and $F^{\prime \prime}$ intersect $Z_{1}$. Then we have

$$
\begin{gathered}
\gamma(D, \mathcal{L})=\Theta_{F, D}=\Theta_{F, D^{\prime}}=f_{D^{\prime}[F]}\left(Z_{1}\right)+f_{D^{\prime}[F]}\left(Z_{2}\right) \geq \\
\geq f_{D^{\prime}[F]}\left(Z_{1}\right) \geq f_{D^{\prime}\left[F^{\prime}\right]}\left(Z_{1} \cap F^{\prime}\right)+f_{D^{\prime}\left[F^{\prime \prime}\right]}\left(Z_{1} \cap F^{\prime \prime}\right)= \\
=\varrho_{D^{\prime}\left[F^{\prime}\right]}\left(Z_{1} \cap F^{\prime}\right)+\varrho_{D^{\prime}\left[F^{\prime \prime}\right]}\left(Z_{1} \cap F^{\prime \prime}\right) \geq \\
\quad \geq \frac{\mu\left(D^{\prime}\left[F^{\prime}\right]\right)}{2}+\frac{\mu\left(D^{\prime}\left[F^{\prime \prime}\right]\right)}{2}>\gamma(D, \mathcal{L})
\end{gathered}
$$

a contradiction. Here the second inequality follows from the definition of the function $f$, the equality following it is because $a_{F^{\prime \prime \prime}} \in Z_{1}$ if $F^{\prime \prime \prime} \in \mathcal{L}_{Z_{1}}$ is a proper subset of $F^{\prime}$ or $F^{\prime \prime}$. The next inequality follows from the definition of $a_{F^{\prime}}$ and $a_{F^{\prime \prime}}$, and the last (strict) inequality is by the minimal choice of $F$.

In the other case, when $F^{\prime}$ intersects $Z_{1}$ and $F^{\prime \prime}$ intersects $Z_{2}$, we get the contradiction in a similar way:

$$
\begin{aligned}
& \gamma(D, \mathcal{L})= \Theta_{F, D}=\Theta_{F, D^{\prime}}=f_{D^{\prime}[F]}\left(Z_{1}\right)+f_{D^{\prime}[F]}\left(Z_{2}\right) \geq \\
& \geq f_{D^{\prime}\left[F^{\prime}\right]}\left(Z_{1} \cap F^{\prime}\right)+f_{D^{\prime}\left[F^{\prime \prime}\right]}\left(Z_{2} \cap F^{\prime \prime}\right)=\varrho_{D^{\prime}\left[F^{\prime}\right]}\left(Z_{1} \cap F^{\prime}\right)+\varrho_{D^{\prime}\left[F^{\prime \prime}\right]}\left(Z_{2} \cap F^{\prime \prime}\right) \geq \\
& \geq \frac{\mu\left(D^{\prime}\left[F^{\prime}\right]\right)}{2}+\frac{\mu\left(D^{\prime}\left[F^{\prime \prime}\right]\right)}{2}>\gamma(D, \mathcal{L})
\end{aligned}
$$

3. Therefore we are left with two cases. In the first case assume that $a_{F^{\prime}} \in Z_{1} \cup Z_{2}$ holds for every $F^{\prime} \in \mathcal{L}_{Z_{1} \cup Z_{2}}$ with $F^{\prime} \subsetneq F$. In that case we have that $f_{D^{\prime}[F]}\left(Z_{i}\right)=\varrho_{D^{\prime}[F]}\left(Z_{i}\right)$ for both $i=1,2$, and thus $\gamma(D, \mathcal{L})=\Theta_{F, D^{\prime}}=\sum_{i=1,2} \varrho_{D^{\prime}[F]}\left(Z_{i}\right) \geq \mu\left(D^{\prime}[F]\right) \geq \Theta_{F, D^{\prime}}$.

4. In our last case there exists a unique inclusionwise minimal $F^{\prime} \in \mathcal{L}_{Z_{1} \cup Z_{2}}$ such that $F^{\prime}$ is a proper subset of $F$ with $a_{F^{\prime}} \notin Z_{1} \cup Z_{2}$. Assume without loss of generality that $F^{\prime}$ intersects $Z_{1}$ and choose an arbitrary $a \in$ $F^{\prime} \cap Z_{1}$. Then $f_{D_{a}[F]}\left(Z_{i}\right)=\varrho_{D_{a}[F]}\left(Z_{i}\right)$ for both $i=1,2$, and thus $\gamma(D, \mathcal{L})=\Theta_{F, D_{a}}=\sum_{i=1,2} \varrho_{D_{a}[F]}\left(Z_{i}\right) \geq$ $\mu\left(D_{a}[F]\right) \geq \Theta_{F, D_{a}}$.

\subsection{Running time}

Let $T(N, M)$ be the time needed to find a minimum $s-t$ cut in an edge-weighted digraph having $N$ nodes and $M \operatorname{arcs}$ (that is, $M \leq N^{2}$ here).

The natural weighted version of Problem 4 is the following.

Problem 5. Given a digraph $D=(V, A)$, and a nonnegative weight function $w: A \rightarrow \mathbb{R}_{+}$, and a laminar family $\mathcal{L} \subseteq 2^{V}$, find a subset $H$ of the arc set such that $H$ intersects every $\mathcal{L}$-tight arborescence and $w(H)$ is minimum.

As mentioned above, if we want to solve the weighted Problem 5 , the only thing to be changed is that the in-degree $\varrho(X)$ of a set should mean the weighted in-degree. We will analyze the algorithm in this sense, so we assume that the input digraph does not contain parallel arcs, but weighted ones.

In order to analyze the performance of Algorithm COVERING_TIGHT_ARBORESCENCES, let $n$ and $m$ denote the number of nodes and arcs in its input (so $m \leq n^{2}$ ).

To implement the algorithm above we need 2 subroutines. The first subroutine finds an anchor node in an edge-weighted digraph. This subroutine will be used $|\mathcal{L}| \leq n$ times in Step 1.4 for digraphs having at most $n$ nodes and at most $m$ arcs. By the definition of anchor nodes, any node $r$ maximizing $\min \left\{\varrho_{G}(X): \emptyset \neq X \subseteq\right.$ $V-r\}$ can serve as an anchor node. Therefore, finding an anchor can be done in $n^{2} T(n, m)$ time .

The second subroutine determines $\mu(G)$ for a given edge-weighted digraph $G$. This subroutine is used at most $n$ times in Step 1.12 and $n^{2}$ times in Step 1.14 for digraphs having at most $n$ nodes and at most $m$ arcs. Note however that these suboutine calls are not independent from each other, and we will make use of this fact later.

We can determine $\mu(G)$ for a given edge-weighted digraph $G$ the following way. Take two disjoint copies of $G$, and reverse all arcs in the first copy (and denote this modified first copy by $G^{1}$ ). Let the second copy 
be denoted by $G^{2}$, and for each $v \in V(G)$ let the corresponding node in $V\left(G^{i}\right)$ be $v^{i}$ for $i=1,2$. For each $v \in V(G)$ add an arc $v^{1} v^{2}$ of infinite capacity from $v^{1}$ to its corresponding copy $v^{2} \in V\left(G^{2}\right)$. This way we define an auxiliary weighted digraph $\hat{G}=\left(V\left(G^{1}\right) \cup V\left(G^{2}\right), A\left(G^{1}\right) \cup A\left(G^{2}\right) \cup\left\{v^{1} v^{2}: v \in V(G)\right\}\right)$. It is easy to see that for some $s \neq t$ nodes in $V(G)$ we have $\min \left\{\delta_{\hat{G}}(Z): s^{1} \in Z \subseteq V(\hat{G})-t^{2}\right\}=\min \left\{\varrho_{G}(X)+\varrho_{G}(Y): s \in\right.$ $X \subsetneq V(G), t \in Y \subsetneq V(G), X \cap Y=\emptyset\}$. Thus, by trying every possible pair $s, t$, we can calculate $\mu(G)$ with $n^{2}$ minimum $s^{1}-t^{2}$-cut computations in $\hat{G}$ in time $n^{2} T(n, m)$.

We will calculate $\mu(G)$ for $O\left(n^{2}\right)$ graphs $G$ (each having at most $n$ nodes and $m$ arcs): $n$ times in Step 1.12 for the graphs $D^{\prime}[F]$, and $n^{2}$ times in Step 1.14 for the graphs $D_{a}[F]$. On the other hand, as mentioned earlier, these calls are not independent from each other, since if $\mu\left(D_{a}[F]\right)<\mu\left(D^{\prime}[F]\right)$ for some $F \in \mathcal{L}$ and $a \in F$ then $a \in X \cup Y$ has to hold for the (optimal disjoint non-empty) sets $X, Y \subseteq F$ giving $\mu\left(D_{a}[F]\right)=\varrho_{D_{a}[F]}(X)+\varrho_{D_{a}[F]}(Y)$. Therefore checking whether $\mu\left(D_{a}[F]\right)<$ best or not in Step 1.14, we only need to calculate minimum $a^{1}-t^{2}-$ cuts in $\widehat{D_{a}[F]}$ (for the node $a^{1} \in V\left(\left(D_{a}[F]\right)^{1}\right)$ corresponding to $a$ and every $t^{2} \in V\left(\left(D_{a}[F]\right)^{2}\right)$ corresponding to nodes $t \in F-a)$, needing only $n$ minimum cut computations.

Putting everything together we get that the Algorithm COVERING_TIGHT_ARBORESCENCES can be implemented to run in $n^{3} T(n, m)$ time. It seems possible to further reduce the complexity of Steps 1.4 and 1.12 however we don't know how to do this for Step 1.14 .

\subsection{Remarks on the polyhedral approach}

The tractability of the weighted Problem 5 is equivalent with optimization over the following polyhedron:

$$
\mathcal{P}:=\operatorname{conv}\left(\left\{\chi_{H}: H \text { a } \mathcal{L} \text {-double cut }\right\}\right)+\mathbb{R}_{+}^{A} .
$$

A completely different approach to the problem would be to directly show that this polyhedron $\mathcal{P}$ is tractable, which hinges upon finding a nice polyhedral description of the given polyhedron. Firstly, the polyhedron has facets with large coefficients, which rules out a rank-inequality type description. Secondly, the polyhedron seems to be of a composite nature in the following sense. For $\mathcal{L}=\emptyset$,

$$
\mathcal{P}=\operatorname{conv}\left(\bigcup_{s \neq t, s, t \in V} \operatorname{conv}\left(\left\{\chi_{H}: H \text { a double cut separating } s, t\right\}\right)+\mathbb{R}_{+}^{A}\right),
$$

where a double cut is said to separate $s, t$ if $s \in Z_{1}, t \in Z_{2}$. Thus optimization over $\mathcal{P}$ reduces to optimization over $\left(\begin{array}{c}n \\ 2\end{array}\right)$ polyhedra of double cuts separating a given pair $s, t$. For any given pair $s, t$, this polyhedron has a nice description, and also nice combinatorial algorithm for optimization. When we apply this approach to a general $\mathcal{L}$, then we need to consider the union of an exponential number of polyhedra: one for every possible choice of an anchor node in every set of $\mathcal{L}$. Thus the proposed approach only results in an efficient algorithm for the special case $\mathcal{L}=\emptyset$, and leaves the general case without a polyhedral description.

\section{$6 \quad$ Further notes}

As we have already pointed out, Problems 1 and 2 can be interpreted as covering minimum cost common bases of two matroids. In fact they can be interpreted as covering common bases of a graphic matroid and a partition matroid, too, as we show below. Let us start with a general observation which states that the problem of covering minimum cost common bases of two matroids can be reduced to the problem of covering common bases of two matroids.

Theorem 6. Given two matroids $M_{1}$ and $M_{2}$ over the same ground set $S$, a cost function $c: S \rightarrow \mathbb{R}$, assume that $M_{1}$ and $M_{2}$ have a common base, then there exist matroids $M_{1}^{\prime}$ and $M_{2}^{\prime}$ over $S$ so that the minimum c-cost common bases of $M_{1}$ and $M_{2}$ are the common bases of $M_{1}^{\prime}$ and $M_{2}^{\prime}$.

Proof. The simplest way to see this is through Frank's Weight Splitting Theorem [6]. It states, that the assumptions of our theorem imply the existence of cost functions $c_{1}, c_{2}: S \rightarrow \mathbb{R}$ so that $c=c_{1}+c_{2}$ and a common base of $M_{1}$ and $M_{2}$ has minimal $c$-cost if and only if it is a $c_{i}$-minimum cost base of $M_{i}$ for both $i=1,2$. As the family $\mathcal{B}_{i}=\left\{B \subseteq S: B\right.$ is a $c_{i}$-minimum cost base of $\left.M_{i}\right\}$ is the family of bases of a matroid $M_{i}^{\prime}$, the theorem follows.

Let us specialize Theorem 6 for our problems. Given a digraph $D=(V, A)$ with a designated node $r \in V$, and a cost function $c: A \rightarrow \mathbb{R}$, as in Problem 1. Fukerson's Theorem (Theorem 1) tells us that we can find in polynomial time a subset $A^{\prime} \subseteq A$ of arcs (tight arcs) and a laminar family $\mathcal{L} \subseteq 2^{V-r}$ such that an $r$-arborescence is of minimum cost if and only if it uses only tight arcs and it is $\mathcal{L}$-tight. Using Claim 1 we get the following: $\{B \subseteq A: B$ is an optimal $r$-arborescence $\}=\left\{B \subseteq A^{\prime}: B[F]\right.$ is a tree spanning $F$ for every $F \in \mathcal{L} \cup\{V\}$ and $\varrho_{B}(v)=1$ if $v \in V-r$ and $\left.\varrho_{B}(r)=0\right\}$. Let us introduce the definition of $\mathcal{L}$-tight spanning trees. 
Definition 5. Given a connected graph $G=(V, E)$ and a family $\mathcal{F} \subseteq 2^{V}$, a spanning tree $B \subseteq E$ is said to be $\mathcal{F}$-tight if $B[F]$ is a tree spanning $F$ for every $F \in \mathcal{F}$.

The following claim is easy to prove.

Claim 11. Given a graph $G=(V, E)$ and a family $\mathcal{F} \subseteq 2^{V}$, define a cost function $c_{1}: E \rightarrow \mathbb{Z}_{+}$as $c_{1}(e)=$ $|\{F \in \mathcal{F}: e \subseteq F\}|$. If there exists a $\mathcal{F}$-tight spanning tree then the $\mathcal{F}$-tight spanning trees are the maximum $c_{1}$-cost spanning trees.

We will need one more observation stating that the matroid of maximum cost spanning trees of a graph is a graphic matroid.

Claim 12. Given a connected graph $G=(V, E)$ and a cost function $c_{1}: E \rightarrow \mathbb{R}$, there exists another graph $G^{\prime}=\left(V^{\prime}, E^{\prime}\right)$ and a bijection $\phi: E \rightarrow E^{\prime}$ so that $T \subseteq E$ is a maximum $c_{1}$-cost spanning tree in $G$ if and only if $\phi(T)$ is an inclusionwise maximal forest in $G^{\prime}$ (that is, a base of the circuit matroid of $G^{\prime}$ ).

Applying Claims 11 and 12 to Problem 1 we get the following theorem.

Theorem 7. Given a digraph $D=(V, A)$ with a designated node $r \in V$, and a cost function $c: A \rightarrow \mathbb{R}$, we can find in polynomial time a subset $A^{\prime} \subseteq A$, a graphic matroid $M_{1}$ and a partition matroid $M_{2}$ (both on ground set $\left.A^{\prime}\right)$ so that $B \subseteq A$ is a minimum c-cost $r$-arborescence of $D$ if and only if $B \subseteq A^{\prime}$ and it is a common base of $M_{1}$ and $M_{2}$.

Thus, the problem of covering optimal $r$-arborescences (Problem 1 ) can be reduced to the problem of covering common bases of a graphic matroid and a partition matroid (with some correlation between these two matroids). Following the reformulations above one can probably work out a polynomial time algorithm (different from ours) for Problem 1, too. This can be a direction for further research.

\section{Acknowledgements}

We thank Naoyuki Kamiyama for calling our attention to this problem at the 7th Hungarian-Japanese Symposium on Discrete Mathematics and Its Applications in Kyoto. We would like to thank Kristóf Bérczi, András Frank, Erika Kovács, Tamás Király and Zoltán Király of the Egerváry Research Group for useful discussions and remarks. A version of this paper was presented at the 21st International Symposium on Mathematical Programming (ISMP 2012) and at the 16th Conference on Integer Programming and Combinatorial Optimization (IPCO 2013).

\section{References}

[1] Mihály Bárász, Johanna Becker, and András Frank, An algorithm for source location in directed graphs, Oper. Res. Lett. 33 (2005), no. 3, 221-230.

[2] Charles J. Colbourn and Ehab S. Elmallah, Reliable assignments of processors to tasks and factoring on matroids, Discrete Mathematics 114 (1993), no. 1-3, 115 - 129.

[3] Gérard Cornuéjols, Combinatorial optimization: Packing and covering, vol. 74, SIAM, 2001.

[4] Michael Dinitz and Anupam Gupta, Packing interdiction and partial covering problems, Integer Programming and Combinatorial Optimization, Springer, 2013, pp. 157-168.

[5] Jack Edmonds, Edge-disjoint branchings, Combinatorial algorithms 9 (1973), 91-96.

[6] András Frank, A weighted matroid intersection algorithm, Journal of Algorithms 2 (1981), no. 4, 328-336.

[7] Greg N Frederickson and Roberto Solis-Oba, Increasing the weight of minimum spanning trees, Journal of Algorithms 33 (1999), no. 2, 244-266.

[8] D. R. Fulkerson, Packing rooted directed cuts in a weighted directed graph, Mathematical Programming 6 (1974), 1-13, 10.1007/BF01580218.

[9] Jim Geelen and Rohan Kapadia, Computing girth and cogirth in perturbed graphic matroids, arXiv preprint arXiv:1504.07647 (2015).

[10] The EGRES Group, Covering minimum cost spanning trees, EGRES QP-2011-08, www.cs.elte.hu/egres.

[11] Gwenaël Joret and Adrian Vetta, Reducing the rank of a matroid, arXiv preprint arXiv:1211.4853 (2012). 
[12] Naoyuki Kamiyama, Robustness of minimum cost arborescences, ISAAC (Takao Asano, Shin-Ichi Nakano, Yoshio Okamoto, and Osamu Watanabe, eds.), Lecture Notes in Computer Science, vol. 7074, Springer, 2011, pp. 130-139.

[13] Tamás Király, Computing the minimum cut in hypergraphic matroids, Tech. Report QP-2009-05, Egerváry Research Group, Budapest, 2009, www.cs.elte.hu/egres.

[14] Weifa Liang and Xiaojun Shen, Finding the $k$ most vital edges in the minimum spanning tree problem, Parallel Computing 23 (1997), no. 13, 1889-1907.

[15] Michel Lorea, Hypergraphes et matroides, Cahiers Centre Etud. Rech. Oper 17 (1975), 289-291.

[16] S Thomas McCormick, A combinatorial approach to some sparse matrix problems., Tech. report, DTIC Document, 1983.

[17] Alexander Schrijver, Combinatorial optimization: polyhedra and efficiency, vol. 24, Springer Verlag, 2003.

[18] Alexander Vardy, The intractability of computing the minimum distance of a code, IEEE Transactions on Information Theory 43 (1997), no. 6, 1757-1766.

[19] Rico Zenklusen, Matching interdiction, Discrete Applied Mathematics 158 (2010), no. 15, $1676-1690$.

[20] Rico Zenklusen, Bernard Ries, Christophe Picouleau, Dominique De Werra, M-C Costa, and Cédric Bentz, Blockers and transversals, Discrete Mathematics 309 (2009), no. 13, 4306-4314. 